\title{
A NEW CLASS OF SYMMETRIC WEIGHING MATRICES
}

\author{
H. KHARAGHANI
}

(Received 18 June 1984)

Communicated by W. D. Wallis

\begin{abstract}
If there is a $W(n, p)$, then there is a symmetric $W\left(n^{2}, p^{2}\right)$.

1980 Mathematics subject classification (Amer. Math. Soc.): 05 B 20.

A weighing matrix of weight $p$ and order $n$ is an $n \times n\{0,1,-1\}$-matrix $A$ such that $A A^{t}=A^{t} A=p I_{n}$. We refer to such a matrix as a $W(n, p)$. $\mathrm{A} W(n, p)$ is called a Hadamard matrix. Goethals and Seidel [2] proved that if there is a Hadamard matrix of order $n$, then there is a symmetric Hadamard matrix of order $n^{2}$. W. D. Wallis [3], partially answering a question of Bush, proved that if there is a Hadamard matrix of order $n$, then there is a symmetric Hadamard matrix of order $n^{2}$ which can be partitioned into an $n \times n$ array of $n \times n$ blocks such that (i) each diagonal block has every entry 1 , and (ii) each non-diagonal block has every row and column sum zero (we will refer to such matrices as Bush-type Hadamard). In this paper, with an entirely new approach, we shall prove that if $A$ is a $W(n, p)$, then $A^{t} \times A$ is Hadamard equivalent to a symmetric $W\left(n^{2}, p^{2}\right)$ (see [4, page 408]). This provides many new symmetric weighing matrices and, with a slight modification, proves the following: if $H$ is a Hadamard matrix of order $n$, then $H^{t} \times H$ is Hadamard equivalent to a Bush-type Hadamard matrix of order $n^{2}$. Throughout the note we will follow Geramita and Seberry [1] for definitions, etc.
\end{abstract}

THEOREM 1. Let $A$ be a $W(n, p)$. Then there is a symmetric $W\left(n^{2}, p^{2}\right)$ which can be obtained from $A^{t} \times A$ by reordering the rows.

(C) 1986 Australian Mathematical Society $0263-6115 / 86 \$ A 2.00+0.00$ 
Proof. Let $A=\left[a_{i j}\right]$. For $k, l=1,2, \ldots, n$, let $C_{k l}=\left[a_{l i} a_{k j}\right]$. Then

$$
C_{l k}^{t}=\left[a_{k i} a_{l j}\right]^{t}=\left[a_{k j} a_{l i}\right]=C_{k l}, \text { for } 1 \leqslant l, k \leqslant n \text {. }
$$

$$
C_{k l} C_{k^{\prime} l}^{\prime}=\left[\sum_{m} a_{l i} a_{k m} a_{k^{\prime} m} a_{l j}\right]=\left[a_{l i}\left(\sum_{m} a_{k m} a_{k^{\prime} m}\right) a_{l j}\right]
$$

$$
= \begin{cases}0 & \text { if } k \neq k^{\prime} \\ p C_{l l} & \text { if } k=k^{\prime}\end{cases}
$$

$$
\sum_{l} C_{k l} C_{k l}^{t}=p \sum_{l} C_{l l}=p\left[\sum_{l} a_{l i} a_{l j}\right]=p^{2} I_{n} .
$$

Consider the block matrix $B=\left[C_{k l}\right], k, l=1,2, \ldots, n$. Then $B$ is a $\{0,1,-1\}$ matrix with the following properties:

$$
\begin{gathered}
B^{t}=\left[C_{l k}^{t}\right]=\left[C_{k l}\right]=B \quad \text { by (i) above; } \\
B B^{t}=\left[\sum_{m} C_{k m} C_{l m}^{t}\right]=p^{2} I_{n^{2}} \quad \text { by (ii) and (iii) above. }
\end{gathered}
$$

Hence $B$ is a symmetric $W\left(n^{2}, p^{2}\right)$.

Finally, it is easy to see that row $1+(i-1) n+j$ of $A^{t} \times A$ is exactly the same as row $i+n j$ of $B$, for $i=1,2, \ldots, n$ and $j=0,1,2, \ldots, n-1$. So $B$ can be obtained from $A^{t} \times A$ by reordering the rows of $A^{t} \times A$.

The following is easier than Goethals and Seidel's original construction.

Corollary 2 (Goethals and Seidel [2]). Let $A$ be a Hadamard matrix. Then there is a symmetric Hadamard matrix with constant diagonal which could be obtained from $A^{t} \times A$ by reordering the rows.

Proof. Let $p=n$ in Theorem 1 and note that all entries on the main diagonal of $B$ are ones.

Actually we have more than the above.

Corollary 3 (W. D. Wallis [3]). Let $A$ be a Hadamard matrix. Then $A^{t} \times A$ is Hadamard equivalent to a Bush-type Hadamard matrix.

Proof. Let $B$ be the matrix constructed in Theorem 1. Multiply the columns of $B$ by the corresponding entries of the first row of $C_{i i}, i=1,2, \ldots, n$, and apply the corresponding row operations. This is like changing $C_{k l}$ in Theorem 1 by $D_{k l}=\left[a_{l i} a_{l j} a_{k i} a_{k j}\right]$ for each $k, l=1,2, \ldots, n$. So the new block matrix $D=\left[D_{k l}\right]$ remains symmetric and Hadamard equivalent to $A^{t} \times A$. Furthermore

$$
\begin{aligned}
& D_{l l}=\left[a_{l i} a_{l j} a_{l i} a_{l j}\right]=[1]=J=\text { the matrix of ones, for each } \\
& l=1,2, \ldots, n,
\end{aligned}
$$


(ii)

$$
\sum_{i} a_{l i} a_{k j} a_{l j} a_{k i}=a_{k j}\left(\sum_{i} a_{l i} a_{k i}\right) a_{l j}= \begin{cases}0 & \text { if } l \neq k \\ n & \text { if } l=k\end{cases}
$$

Similarly,

$$
\sum_{j} a_{l i} a_{k j} a_{l j} a_{k i}= \begin{cases}0 & \text { if } l \neq k, \\ n & \text { if } l=k .\end{cases}
$$

Consequently $D_{k l} J=J D_{k l}=\delta_{l k} n J$. Hence $D$ is Bush-type Hadamard. Note that each of the blocks in $D$ is symmetric.

To the best of our knowledge the existence of a symmetric $W\left(n^{2}, p^{2}\right)$, as proved here, is new. We refer the reader to [1, pages 215-217] for more information on symmetric weighing matrices and their applications.

\section{References}

[1] A. V. Geramita and Jennifer Seberry, Orthogonal designs, quadratic forms and Hadamard matrices (Lecture Notes in Pure and Applied Mathematics, Vol. 45, Marcel Dekker, New York and Basel, 1979).

[2] J. M. Goethals and J. J. Seidel, 'Strongly regular graphs derived from combinatorial designs', Canad. J. Math. 22 (1970), 597-614.

[3] W. D. Wallis, 'On a problem of K. A. Bush concerning Hadamard matrices', Bull. Austral. Math. Soc. 6 (1972), 321-326.

[4] W. D. Wallis, Anne Penfold Street and Jennifer Seberry Wallis, Combinatorics: Room squares, sum-free sets, Hadamard matrices (Lecture Notes in Mathematics, Vol. 272, Springer-Verlag, Berlin-Heidelberg-New York, 1972).

\section{Department of Mathematics}

University of Alberta

Edmonton, Alberta

Canada

T6G 2G1 